\title{
Perfil de recém-natos com falta de selamento labial e presença de respiração mista na região sul do Tocantins, Brasil
}

\section{Profile of newborns with lack of lip sealing and presence of mixed breathing in the southern region of Tocantins, Brazil}

Sthefane Simão Sousa, ${ }^{1}$ Fernanda Karoline Arruda Pamplona, ${ }^{2}$ Walmirton Bezerra D'Alessandro, ${ }^{3}$ Joana Estela Vilela , ${ }^{4}$ Rise Consolação luata Costa Rank $^{5}$

\section{RESUMO}

Introdução: A função respiratória é fundamental para o ser humano e é a primeira estabelecida ao nascimento. A respiração mista é caracterizada por respiração bucal e nasal, e pode ocorrer por obstrução nasal ou hábito adquirido. Objetivo: Este estudo tem o objetivo de verificar o perfil dos recém-nascidos com presença de respiração mista avaliados na maternidade do Hospital referência na região Sul do Estado do Tocantins, Brasil. Metodologia: Trata-se de um estudo retrospectivo documental do Programa de Promoção em Saúde bucal "Boquinha do Bebê", que realizou levantamento de dados de natureza quantitativa em 1.198 recém-nascidos, em um total de 198 visitas no Hospital Materno Infantil de Gurupi nos anos de 2017 a 2019. Resultados: Da amostra total, encontrou-se $5 \%(n=60)$ destas crianças com falta de selamento labial, com respiração mista. Suas mães tinham a idade média de $24 \pm 5,87$. De todas as crianças examinadas, apenas $5 \%$ tinham alguma obstrução nasal. A língua, em geral (92\%), apresentou postura incorreta com posição inferior para facilitar a entrada de ar pela boca. A maioria das mães (95\%) não percebeu que a criança estava com falta de selamento labial, respirando pela boca. Conclusão: Conclui-se que é importante levantar dados epidemiológicos das alterações respiratórias de forma precoce, e os dados deste estudo demonstraram que os pais, ao nascimento de seu filho, não percebem estas alterações bucais, podendo estabelecer uma disfunção que poderá provocar vários distúrbios na saúde desta criança, caso não seja realizado alguma intervenção.

\section{Palavras-chave: Recém-nascido, Respiração bucal, má oclusão, Desenvolvimento infantil.}

\section{ABSTRACT}

Introduction: Respiratory function is fundamental for humans and is the first established at birth. Mixed breathing is characterized by mouth and nasal breathing, and may occur due to nasal obstruction or acquired habit. Objective: This study aims to verify the profile of newborns with mixed breathing in those evaluated at the maternity hospital of the reference hospital in the southern region of the State of Tocantins, Brazil. Methodology: This is a retrospective documentary study of the Oral Health Promotion Program "Boquinha do Bebê", which conducted quantitative data collection in 1,198 newborns, in a total of 198 visits to the Maternal Infant Hospital of Gurupi from 2017 to 2019. Results: From the total sample, 5\% $(n=60)$ of these children with a lack of lip sealing with mixed breathing were found. Their mothers had a mean age of $24 \pm 5.87$. Of all the children examined, only $5 \%$ had some nasal obstruction. The language in general $(92 \%)$, presented incorrect posture with a lower position to facilitate the entry of air through the mouth. Most mothers (95\%) did not notice that the child was lacking lip sealing, breathing through the mouth. Conclusion: It is concluded that it is important to collect epidemiological data of respiratory alterations early, and the data of this study demonstrated that the parents, at the birth of their child, do not perceive these oral alterations, and may establish a dysfunction that may cause several disorders in the health of this child, if no intervention is performed.

Keywords: Newborn, Mouth breathing, Malocclusion, Child development

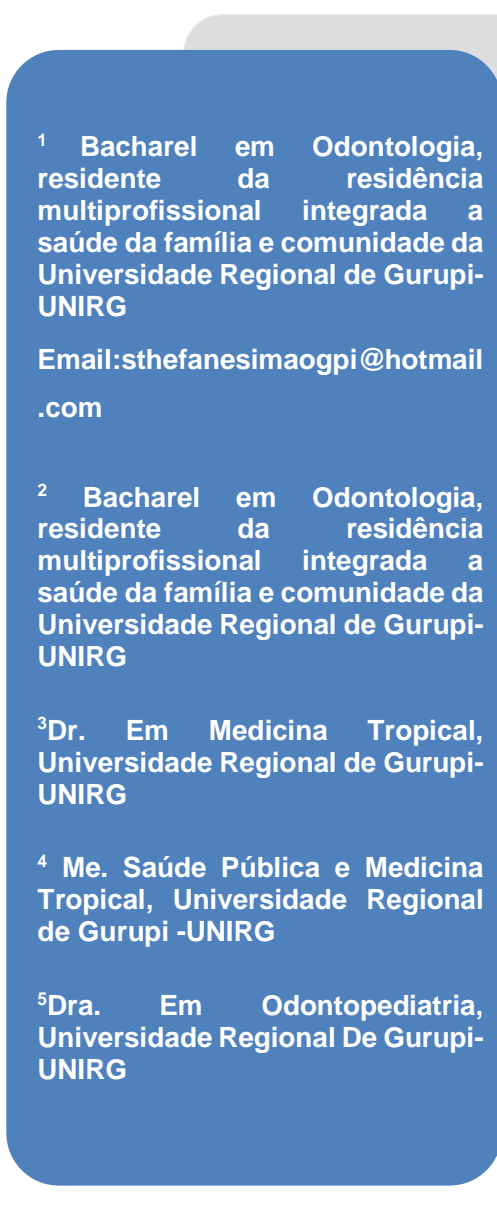

${ }^{3}$ Dr. Em Medicina Tropical, Universidade Regional de GurupiUNIRG

${ }^{4}$ Me. Saúde Pública e Medicina Tropical, Universidade Regional de Gurupi -UNIRG

${ }^{5}$ Dra. Em Odontopediatria, Universidade Regional De GurupiUNIRG 


\section{INTRODUCÁAO}

A respiração surge como um reflexo neuromuscular congênito não condicionado que auxilia na manutenção da vida, atua no crescimento e desenvolvimento adequado do complexo craniofacial do indivíduo e para o no funcionamento das demais estruturas estomatognáticas. A partir do crescimento e desenvolvimento do recém-nascido, reflexos condicionados, desejáveis ou não, mediados pela influência do ambiente, vão determinar o estabelecimento do padrão respiratório infantil. A respiração nasal está vinculada a atividades normais de mastigação, deglutição, postura da língua e lábios, além de promover adequada ação muscular, que determinará o crescimento facial e o desenvolvimento ósseo. ${ }^{1}$

A respiração oral favorece alterações em vários órgãos e sistemas, comprometendo a qualidade de vida, interferindo também no crescimento comum da face. Na fisiologia respiratória, o caminho do ar pelas fossas nasais é o mecanismo que proporciona a função normal do sistema respiratório, enquanto a passagem de ar pela boca é adquirida como forma de compensação, encontrando-se como um reflexo condicionado indesejável. ${ }^{2}$

A síndrome do respirador bucal, também conhecida por síndrome da face longa, caracteriza-se por um conjunto de sinais e sintomas característicos de indivíduos que respiram parcial ou totalmente pela boca. O respirador bucal vai possuir predileção por comidas pastosas, se alimentam de boca aberta, apresentam sialorréia noturna, manifesta comportamento agitado, inquieto, irritado, dificuldade de concentração, podendo apresentar queda no rendimento escolar e pouca predisposição para atividades esportivas. ${ }^{3}$

Pacientes respiradores bucais podem apresentar respiração nasal em graus variáveis, ou acontecer dos indivíduos que respirarem pela boca não apresentar nenhuma obstrução das vias aéreas. O diagnóstico deve ser realizado com base no histórico médico, na investigação de alergias e na presença ou não de hipertrofia das tonsilas palatinas. Depois de investigados os fatores causais alguns pacientes podem ser favorecidos com as cirurgias de adenoidectomia ou tonsilectomia. ${ }^{2}$

As problemáticas relacionadas com a respiração bucal são várias e são conhecidas diversas consequências na saúde humana, contudo as causas primárias da respiração bucal são pouco esclarecidas. Este estudo tem o objetivo de verificar o perfil dos recémnascidos com presença de respiração mista avaliados na maternidade do Hospital referência na região Sul do Estado do Tocantins, Brasil. 


\section{MATERIAIS E METODOS}

Trata-se de um estudo retrospectivo documental, com levantamento de dados de natureza quantitativa. Foram realizadas 198 visitas na maternidade do Hospital Regional de Gurupi, hospital referência do Sul do Tocantins, com avaliação de 1.198 recém-nascidos, diagnosticadas 60 delas com respiração mista.

O programa Boquinha do Bebê atua em Gurupi-TO, nas Unidades Básicas de Saúde municipal, bem como na Maternidade do Hospital de Referência da região Sul do Tocantins, realizando atendimentos infantis para promoção de saúde bucal. Foram examinados recém-nascidos, por meio de visitas, do ano de 2017 a 2019. As que eram constatadas como respiradores mistos, realizava-se o preenchimento de uma ficha avaliativa própria para verificar o perfil destas crianças. Esta pesquisa tem o projeto aprovado pelo Comitê de Ética em Pesquisa/Universidade de Gurupi UnirG com o número do parecer 3.6578 .743 CAAE: 19988619.2.0000.5518.

A pesquisa respeitou todas as diretrizes e regime de acordo com a resolução no 466/12 (BRASIL, 2012), desta forma esta pesquisa apresentou riscos mínimos aos seus participantes, pois não houve exame direto. Os prontuários com as avaliações das crianças com respiração mista foram analisados e os dados encontrados foram tabulados no Programa Excel a análise dos dados foi realizada de forma descritiva, com apresentação em forma de tabela.

\section{RESULTADOS}

Tabela 1: Distribuição em número e percentual dos dados encontrados nos recémnascidos da maternidade do Hospital referência na região Sul do Estado do Tocantins, Brasil (2017-2019).

\begin{tabular}{r|cc}
\hline \multicolumn{2}{l}{ Idade da mãe } & \multicolumn{2}{c}{$24,3 \pm 5,87$} \\
\hline Tipo de parto & $\mathrm{N}$ & $\%$ \\
Normal & 23 & 38.3 \\
Cesáreo & 37 & 61.6 \\
Período de visita & & \\
Manhã & 38 & 63.3 \\
Tarde & 18 & 30
\end{tabular}




\begin{tabular}{|c|c|c|}
\hline Noite & 4 & 6.6 \\
\hline \multicolumn{3}{|l|}{ Bebê estava } \\
\hline Dormindo & 49 & 81 \\
\hline Acordado & 11 & 19 \\
\hline \multicolumn{3}{|l|}{ Posição do bebê* } \\
\hline$D D$ & 21 & 35 \\
\hline$D V$ & 1 & 1.6 \\
\hline$D L$ & 38 & 63.3 \\
\hline \multicolumn{3}{|l|}{ Lábios do bebê } \\
\hline Entreaberto & 56 & 93.3 \\
\hline Aberto & 4 & 6.6 \\
\hline \multicolumn{3}{|l|}{$\begin{array}{r}\text { Interposição da } \\
\text { língua entre lábios }\end{array}$} \\
\hline Sim & 5 & 8,3 \\
\hline Não & 55 & 91,6 \\
\hline \multicolumn{3}{|l|}{$\begin{array}{r}\text { Bebê fica o tempo } \\
\text { todo com essa } \\
\text { postura }\end{array}$} \\
\hline Sim & 3 & 5 \\
\hline Não & 42 & 70 \\
\hline Ás vezes & 13 & 21,6 \\
\hline Não Sei & 2 & \\
\hline \multicolumn{3}{|l|}{$\begin{array}{r}\text { Criança tem } \\
\text { dificuldade de } \\
\text { respirar pelo nariz }\end{array}$} \\
\hline Sim & 3 & 5 \\
\hline Não & 52 & 86,6 \\
\hline Ás vezes & 4 & 6,6 \\
\hline Não Sei & 1 & 1,6 \\
\hline
\end{tabular}

${ }^{*} D D=$ Decúbito dorsal (Posição deitada com a barriga voltada para cima); $D V=$ Decúbito Ventral (Posição Deitada de bruços); $D L=$ Decúbito Lateral (Posição esquerdo ou direito).

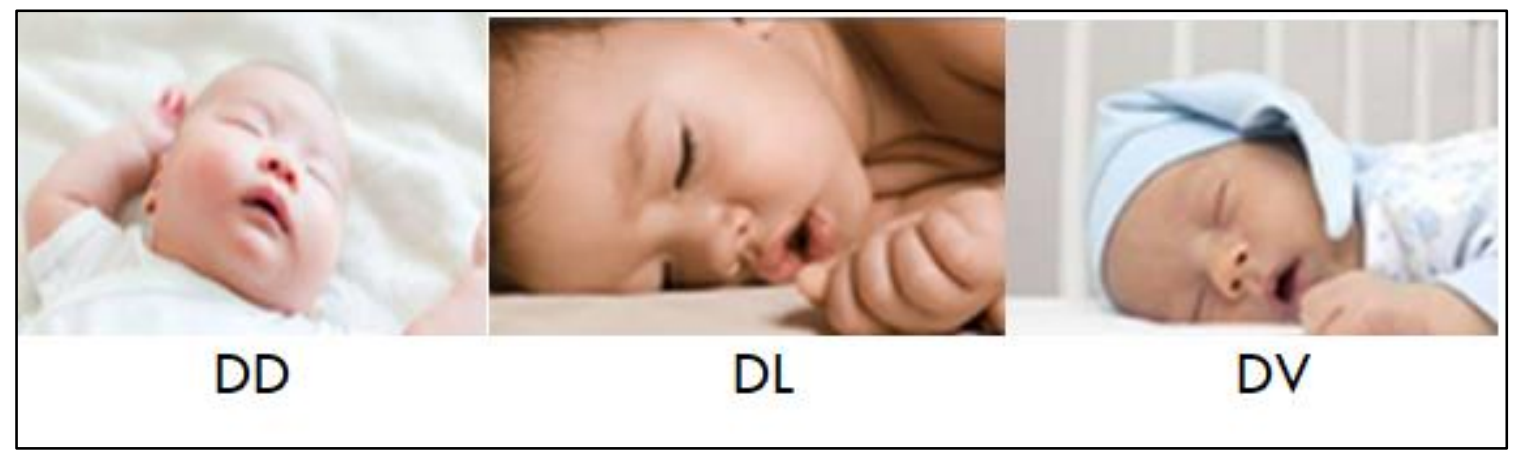

Fonte: https://paisefilhos.uol.com.br/ 
Figura 1: As imagens mostram as posições em que os bebês se encontravam: decúbito Dorsal (DD); decúbito lateral (DL) e Decúbito Ventral (DV).

Dos 1.198 recém-nascidos analisados, foram diagnosticadas 60 delas com respiração mista. A média de idade das mães foi de 24 anos, em que sua maioria recebeu parto cesariano, conforme os dados da maternidade. Ao se examinar, a maioria das crianças estava dormindo em decúbito lateral.

O Tipo de postura da língua, apresentou-se em geral, com posição inferior para facilitar a entrada de ar pela boca e algumas vezes pode-se observá-la com o dorso elevado para regular o fluxo de ar. Desta forma, a língua não foi encontrada com a postura correta, com a ponta na região ântero superior do palato.

Estas crianças com falta de Selamento labial, em sua maioria estavam com os lábios entreabertos e apenas $5 \%$ com a boca aberto.

Sendo o DL o maior encontrado. Mas foi observado também a alta taxa de DD.

Notou-se a falta de percepção das mães, pois quase todas não perceberam que a criança estava com a falta de selamento labial, respirando pela boca, apenas $5 \%$ delas relataram ter observado.

\section{DISCUSSÃO}

O parto normal é o método natural do bebê nascer, sendo que $92 \%$ das gestantes que optam pelo parto normal terão seus bebês sem nenhum problema, com uma recuperação imediata, pois logo após o parto elas podem se levantar e dar as primeiras mamadas ao recém-nascido. Quando comparado às complicações que os partos cesáreos podem promover, a mãe consegue amamentar o bebê de forma mais fácil e prazerosa, e as infecções hospitalares são menos frequentes de ocorrer. Inúmeros são os pontos positivos do parto natural. ${ }^{4}$ A Organização Mundial da Saúde (OMS) considera que os partos cesáreos deveriam ocorrer entre $10 \%$ a $15 \%$ dentre o total, no entanto, estes partos estão cada vez mais adotados em diversos países. Sabe-se que as cesarianas podem reduzir a mortalidade e morbidade materna e perinatal, mas torna-se importante destacar que toda e qualquer cirurgia pode acarretar riscos ou sequelas na mulher ou na criança. No presente estudo observou-se que as taxas de parto cesárea nesta população investigada atingiram $61,6 \%$, valor muito acima do que se preconiza pela OMS, o que motiva a investigação e 
discussão no aspecto das causas que levam aos profissionais buscarem esta intervenção cirúrgica em alta escala, ou se podem ser motivo de causar falta de selamento labial, e consequentemente a disfunção respiratória. ${ }^{5}$

$\mathrm{Na}$ avaliação dos bebês, para observar a posição correta da sua cavidade bucal e respiração, a posição que o bebê estava deitado no berço foi um ponto importante para o estudo. O comportamento durante o sono do bebê, a postura dos olhos, se possuía tremor do mento ou não, os movimentos de sucção que ele fazia ou não com a língua durante o sono e se ele fica com os lábios abertos ou entreabertos são fundamentais para observação. Durante o sono, a criança está com sua musculatura relaxada, e torna-se possível observar o equilíbrio e desiquilíbrio das funções naturais musculares e neurológicas. Segundo Magalhães e Maturana ${ }^{6}$, em seu livro medicina da noite: da cronobiologia à prática clínica, descrevem que antigamente pensava-se que o sono era somente uma parte inativa da vida do ser humano. Hoje em dia, os estudos se avançaram e é comprovado que o cérebro se mantém muito ativo enquanto se está dormindo. Eles classificam o sono, como um estado de diminuição da consciência, que reduz a musculatura, e faz com o que o metabolismo se reduza. Diante disso, as avaliações deste estudo demostram que a maioria da amostra estava dormindo, em que o examinador conseguiu detectar a postura natural e relaxada do participante, mostrando quais deles haviam falta do selamento labial.

A posição que o bebê dorme é de grande importância para a posição dos lábios e postura da boca, além de evitar riscos de sufocamento para o recém-nascido. De acordo com as novas recomendações da Sociedade Brasileira de Pediatria ${ }^{7}$, as recomendações são que o recém-nascido deve dormir em posição de Decúbito Ventral (DV) até um ano de idade, evitando assim a Síndrome da Morte Súbita no Lactente (SMSL). A posição de Decúbito Lateral ( $D L)$ não é considerada segura, pois é uma posição instável e o bebê pode virar e ficar de barriga para baixo, posição que é considerada perigosa. A posição de DV, reduz $70 \%$ de morte súbita do lactante, e caso a criança vomite, consegue tossir e chamar a atenção dos pais. ${ }^{8}$ Os dados da presente pesquisa revelam $63,3 \%$ das crianças avaliadas estavam em $D L$ posição que não é recomendada. A menor taxa $(1,67 \%)$ de recém-nascidos dormia de DV, que é a posição correta. Esses dados remetem um alerta para que a maternidade acompanhe as crianças em seus leitos, e orientem as mães com as recomendações para se evitar complicações aos recém-nascidos. 
De acordo com Silva et al. ${ }^{9}$, muitos respiradores orais tendem a adquirir esta disfunção pelo fator hábito adquirido, não apresentando nenhuma obstrução nasal, ou alteração morfológica anatômica. A característica de um respirador bucal, possui lábios entre abertos. Conforme Tomé, Marchioni, ${ }^{10}$ essa postura dos lábios é justificada por ter uma atividade muscular menor dos músculos orbiculares superior e inferior nos respiradores bucais quando comparados aos nasais. A posição que a língua se encontra na cavidade bucal, pode estar envolvida diretamente com a passagem do ar, para melhorar a respiração. Se a língua está atrás do assoalho da boca, isso impede que o ar circule corretamente, deformando a abobada palatina do bebê. ${ }^{11}$ Este estudo mostrou que a posição da língua da maioria das crianças, mesmo recém-nascidas já estavam em posição incorreta, atrás e abaixo do assoalho da boca, para permitir a passagem da corrente de ar através da boca aberta.

As mães, por desconhecerem a importância da respiração correta nas crianças desde o nascimento, não percebem a falta de selamento labial e a possibilidade de início de uma disfunção respiratória inicial. Esta análise demonstrou que a maioria das mães não perceberam ou sabiam destes cuidados respiratórios.

A pesquisa realizada no banco de dados do programa Boquinha do Bebê, em Gurupi, proporcionou importante levantamento de dados para se conhecer sobre a quantidade de crianças que podem adquirir precocemente esta disfunção respiratória e alertar os profissionais a respeito da possibilidade de prevenir esta alteração na área da pediatria. Por isso, estudos desta disfunção em crianças devem ser mais investigada, para permitir conhecimento dos possíveis fatores e terapêuticas precoces que possam evitar sequelas na comunidade.

\section{CONSIDERAÇÖES FINAIS}

Conclui-se que é importante levantar dados epidemiológicos e perfil da comunidade com alterações respiratórias de forma precoce, pois ainda na maternidade, crianças recémnascidas são encontradas com falta de selamento labial e respiração mista, em que a maioria delas não apresentava obstrução nasal e muitas estavam com a postura incorreta no repouso da língua. Dentre as mães, a maioria delas não percebia que a criança estava com falta de selamento labial, respirando pela boca, podendo estabelecer uma disfunção que poderá provocar vários distúrbios na saúde desta criança, caso não seja realizado alguma intervenção. 


\section{REFERÉNCIAS}

1.Neto ETS, Oliveira AE, Barbosa RW, Oliveira AE, Zandonade E. Fatores associados ao surgimento da respiração bucal nos primeiros meses do desenvolvimento infantil. Rev Bras Crescimento Desenvolvimento Humano. 2009; 19(2):237-248.

2.Silva RZO. O paciente respirador oral e o tratamento ortodôntico [monografia]. Faculdade de Odontologia da Universidade Federal do Rio Grande do Sul Porto Alegre, 2011.

3.Rodrigues SFS. Respiração bucal: implicações biológicas, fisiológicas e ortopédicas [Monografia]. Universidade Fernando Pessoa, Faculdade de Ciências da Saúde. 2014.

4.Ferreira KM, Viana, LV M, Mesquita, MASB. Humanização do Parto normal: uma revisão de literatura, Rev. Saúde em Foco, Teresina, v. 1, n. 2, art. 1, p. 134-148, ago/ dez. 2014.

5.Velho MB, Santos EKA, Colhaço VS. Parto normal e cesárea: representações sociais de mulheres que os vivenciaram. Rev. Bras Enfermagem. 2014. mar-abr; 67(2): 282-289.

6.MAgalhães F, and Mataruna J. Sono. In: JANSEN, JM., et al., orgs. Medicina da noite: da cronobiologia à prática clínica [online]. Rio de Janeiro: Editora Fiocruz, 2007. pp. 103120.

7.Sociedade Brasileira de Pediatria. [ Acesso em 13 de Agosto de 2020]. Disponível em : https://www.sbp.com.br/.

8.Revista pastoral da criança. Ano $3 \cdot \mathrm{N}^{\circ} 09$ agosto -setembro - outubro / 2017. [Acesso em 14 de agosto de 2020]. Disponível em: www.pastoraldacriança.org.br.

9.Silva MAA, Irene QM, Léslie PF, Rafaela S, Rossana RR. Postura, tônus e mobilidade de lábios e língua de crianças respiradoras orais. Rev. CEFAC. 2012 Set-Out; 14(5):853860.

10.Tomé MC, Marchioni SC. Análise eletromagnética dos músculos orbiculares superior e inferior da boca em crianças respiradoras nasais bucais durante a emissão de sílabas. Ver. Pró-fono 1999; 11(1):1-7.

11.Krakauer LH, Guilherme A. Relationship between mouth breathing and postural alterations of children: a descriptive analysis. International Journal of Orofacial Myology. 2000; XXVI:13-23. 